\title{
An improved scatter search algorithm for parameter estimation in large-scale kinetic models of biochemical systems
}

\begin{abstract}
Background: Mathematical models play a central role in facilitating researchers to better understand and comprehensively analyze various processes in biochemical systems. Their usage is beneficial in metabolic engineering as they help predict and improve desired products. However, one of the primary challenges in model building is parameter estimation. It is the process to find nearoptimal values of kinetic parameters which may culminate in the best fit of model prediction to experimental data.

Methods: This paper proposes an improved scatter search algorithm to address the challenging parameter estimation problem. The improved algorithm is based on hybridization of quasi opposition-based learning in enhanced scatter search (QOBLESS) method. The algorithm is tested using a large-scale metabolic model of Chinese Hamster Ovary (CHO) cells.

Results: The experimental result shows that the proposed algorithm performs better than other algorithms in terms of convergence speed and the minimum value of the objective function (loglikelihood). The estimated parameters from the experiment produce a better model by means of obtaining a reasonable good fit of model prediction to the experimental data.

Conclusion: The kinetic parameters' value obtained from our work was able to result in a reasonable best fit of model prediction to the experimental data, which contributes to a better understanding and produced more accurate model. Based on the results, the QOBLESS method can be used as an efficient parameter estimation method in large-scale kinetic model building.
\end{abstract}

Keyword: Systems biology; Scatter search; Opposition-based learning; Bioinformatics; Artificial intelligence; Metabolic engineering 